\title{
Some aspects of hypersonic flow over power law bodies
}

\author{
By H. G. HORNUNG \\ Department of Physics, The Australian National University, Canberra
}

(Received 19 January 1969)

This study concerns the hypersonic flow over blunt bodies in two specific cases. The first is the case when the Mach number is infinite and the ratio of the specific heats approaches one. This is sometimes referred to as the 'Newtonian limit'. The second is the case of infinite Mach number and very large streamwise distance from the blunt nose with a strong shock wave, or the 'blast wave limit'. In both cases attention is restricted to power law bodies. Experiments are described of such flows at $M_{\infty}=7.55$ in air.

The Newtonian flow over bodies of the shape $y \propto x^{m}$ at zero incidence is shown to be divisible into three regions: the attached layer at small $x$, the free layer and the blast wave region. As $m$ increases from zero, the free-layer region reduces in extent until it disappears at $m=1 /(2+j)(j=1$ and 0 for axisymmetric and plane flow respectively). A difficulty arises in a transition solution of the type given by Freeman (1962b) connecting the free layer with the blast wave result. At $m>2 /(3+j)$ the attached layer merges smoothly into the Lees-Kubota solution which replaces the blast-wave result in this range.

In the blast wave limit, solutions were obtained for flow over axisymmetric power law shapes in the range $1 / 2 \gamma<m<\frac{1}{2}$. Second-order results taking account of the body shape are given. These solutions are compared with experimental results obtained in air at a free stream Mach number of 7.55 and stagnation temperature of $630^{\circ} \mathrm{K}$, as well as with numerical solutions at Mach number of 100. The numerical method is tested by comparing solutions corresponding to the experimental conditions with experiment.

\section{The Newtonian limit}

\section{(a) Introduction}

On the front part of a blunt body the Newtonian limit gives a particularly simple first-order solution. The density behind the shock wave is very large, the layer between body and shock is thin, the velocity is constant along streamlines and the pressure field is determined by the rise across the shock, and the subsequent drop across the flow due to streamline curvature. Depending on the body shape this can give negative pressures, which are avoided by assuming that the layer separates, carrying all the mass flow and having zero pressure behind it (Hayes \& Probstein 1966; Lighthill 1957). The shape of such a free layer is thus independent of the body shape downstream of the detachment point. Consideration of higher-order terms (Freeman 1960) shows that this is in fact what 
happens except that the pressure does not fall to zero but to a higher order in $(\gamma-1) /(\gamma+1)=\epsilon$. At larger distances downstream, the free-layer solution breaks down to a transition to the blast wave result which must apply at downstream infinity for slender bodies (Freeman 1962b). The solution given by Freeman $(1962 b)$ is suitable for a general body shape downstream of the detachment point, provided that the blast wave solution applies for the particular shape at $x \rightarrow \infty$.

In the case of power law bodies, $y=x^{m}$, the blast wave solution applies to a first approximation when $m<2 /(3+j),(j=0$ for plane, $j=1$ for axisymmetric flow). Above this value the first-order solution of small-disturbance theory is that given by Lees \& Kubota (1957). Since Freeman's transition solution requires both a detachment point and the applicability of the blast wave result at downstream infinity the question arises as to whether detachment and a blast wave occur in the same range of powers only. It appears that this is not the case (Hornung 1967b) but that there is a value of $m$, below which the Newtonian layer detaches at some finite $x$, and above which it remains attached. This 'limiting detachment shape', $m=1 /(2+j)$, does not correspond to the 'limiting blast wave shape' $m=2 /(3+j)$. As will be seen, the analysis of Freeman (1962b) applies directly for the case $m<1 /(2+j)$ except for a singularity which arises from the breakdown of the assumptions of the Newtonian limit in the stagnation region. Also, for $m>2 /(3+j)$ the transition to the Lees-Kubota solution is direct, but for $2 /(3+j)>m>1 /(2+j)$ a transition solution from the attached Newtonian layer to the blast wave solution could not be obtained.

\section{(b) Pressure distribution and detachment point}

In the approximation $M_{\infty}=\infty, \epsilon \rightarrow 0$ the equations of motion reduce to the following, when higher terms in $\epsilon$ are neglected.

$$
\begin{gathered}
\frac{p(x, \psi)}{\rho_{\infty} U_{\infty}^{2}}=\sin ^{2} \Phi_{s}(x)+\frac{\kappa(x)}{y_{b}^{j}(x)} \int_{\psi s}^{\psi} \cos \Phi_{1}(\psi) \frac{d \psi}{\rho_{\infty} U_{\infty}} \\
\frac{u(\psi)}{\bar{U}_{\infty}}=\cos \Phi_{1}(\psi) \\
\frac{\rho(x, \psi)}{\rho_{\infty}}=\epsilon^{-1} \frac{p(x, \psi)}{\rho_{\infty} U_{\infty}^{2} \sin ^{2} \Phi_{1}(\psi)} \\
z(x, \psi)=\frac{\epsilon}{y_{b}^{j}(x)} \int_{0}^{\psi} \frac{d \psi}{\rho u}
\end{gathered}
$$

where the flow variables have their usual significance. A distorted sketch (figure 1) shows the co-ordinate system $(x, \psi)$ and explains the significance of the suffixes $b, s$ and 1 corresponding to values on the body, shock wave and the point where the streamline $\psi=$ const. crosses the shock wave. $\psi$ is the stream function and $z$ is distance measured normal to the body surface. $\kappa$ is the body curvature.

On a power law body of the shape $y / d=(x / d)^{m}$ where $d$ is the characteristic length scale of the body, it is convenient to introduce the variables $\eta=\cos ^{2} \Phi$ 
and $\eta_{1}=\cos ^{2} \Phi_{1}$. Equation (1) then becomes

where

$$
\frac{p\left(\eta_{1}, \eta\right)}{\rho_{\infty} U_{\infty}^{2}}=1-\eta-\frac{1}{2} \frac{(1-\eta)^{k}}{\eta^{k-\frac{3}{2}}} \int_{\eta_{1}}^{\eta} \frac{\theta^{k-\frac{3}{2}}}{(1-\theta)^{k}} d \theta,
$$

The integral in (5) can be evaluated directly for half-integer or integer values of $k$ and as incomplete beta functions otherwise. The pressure distribution on the body surface $\left(\eta_{1}=0\right)$ is shown in figure 2 in which the values of $m$ correspond to

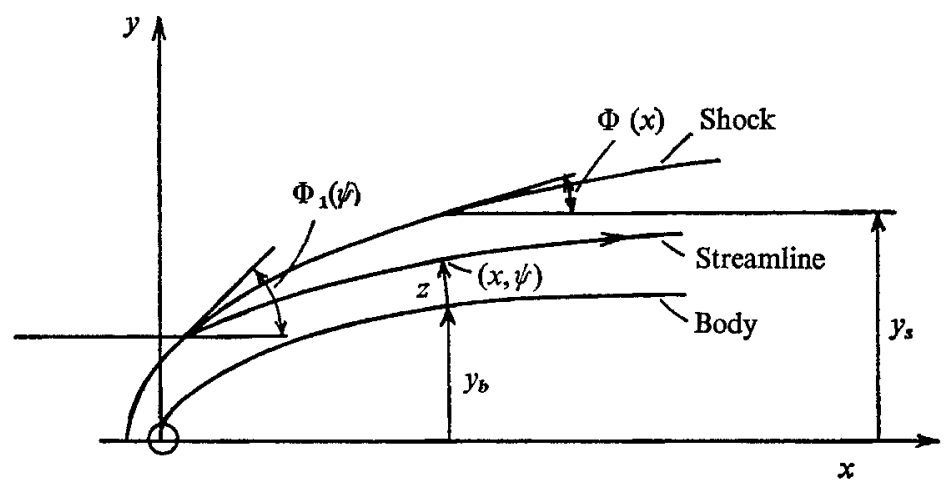

FIgURE 1. Co-ordinates for Newtonian flow.

axisymmetric flow. It is seen that the pressure falls to zero at values of $\eta$ which are different from 1 , only for some values of $m$. That is, there exists a 'limiting detachment shape', which is seen to occur at $k=\frac{3}{2}$ in figure 3 , which shows the value of $\eta$ at detachment, $\eta_{0}$, plotted against $k$. This value of $k=\frac{3}{2}$ corresponds to $m=1 /(2+j)$, such that there is a region of values of $m$, namely $1 /(2+j)<m<2 /(3+j)$ for which no 'free layer' occurs, but where the flow approaches the blast wave solution at large $x$.

\section{(c) Transition to the blast wave solution}

The free layer shape is given, for large $x$, by

$$
y_{s}=c x^{1 /(2+j)},
$$

where $c$ is a constant (see e.g. Hayes \& Probstein 1966).

The shock shape in the blast wave limit is

$$
y_{s} \propto \epsilon^{1 /(3+j)} x^{2 /(3+j)} \text {. }
$$

These two forms can be incorporated in a single transition equation (Freeman $1962 b$ )

$$
y_{s}-\epsilon^{-1 /(2+i)} f\left(x \epsilon^{(2+j) /(1+j)}\right),
$$

where $f(t)$ is a function which must behave like $t^{1 /(2+j)}$ for small $t$ and like $t^{2 /(3+j)}$ for large $t$.

This function can be determined in a closed form from the equation

$$
f\left(f^{1+j} f^{\prime}\right)^{\prime}=\beta
$$

due to Freeman (see also Hayes \& Probstein 1966, p. 377). 


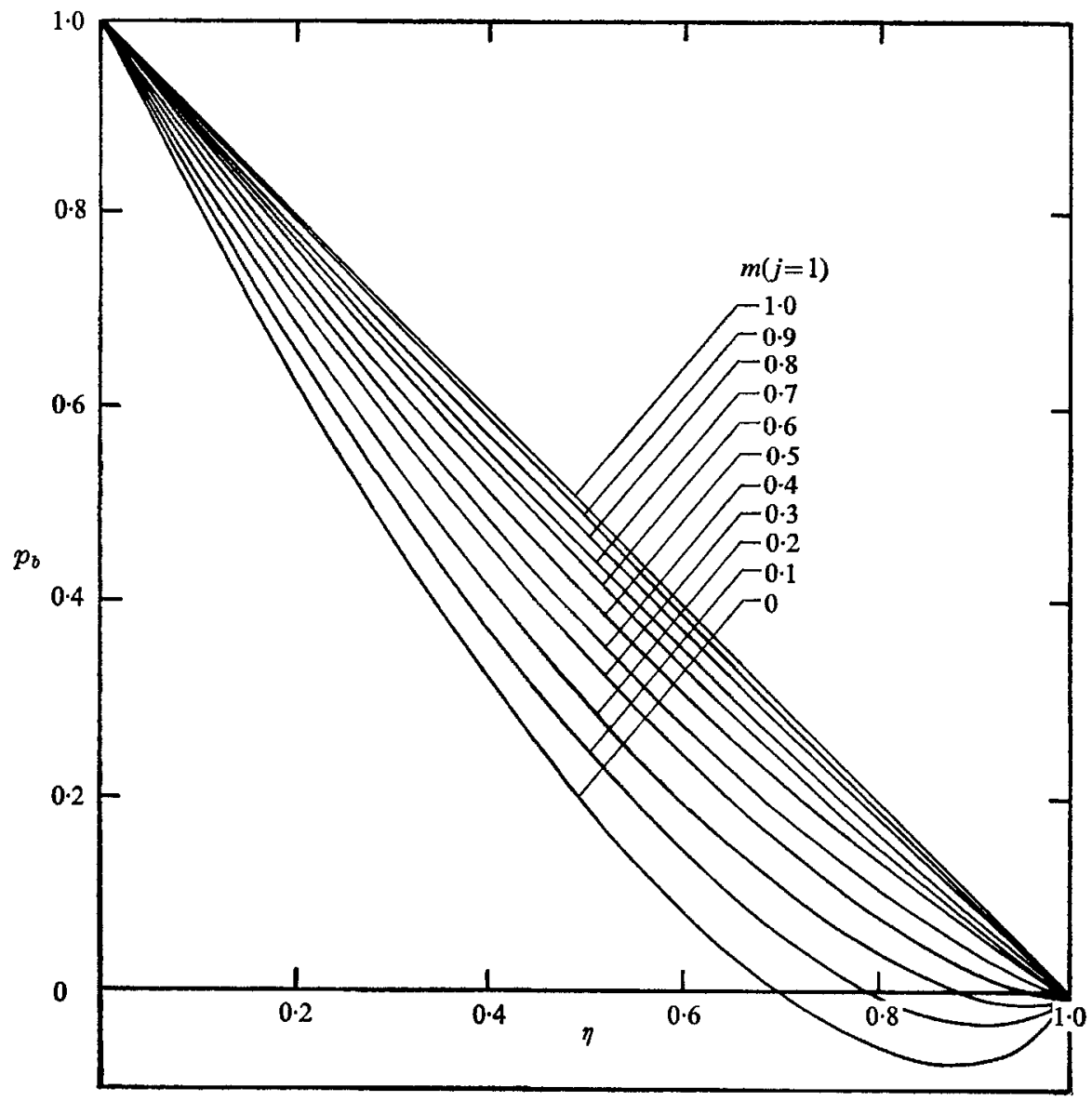

Figure 2. Surface pressure distribution.

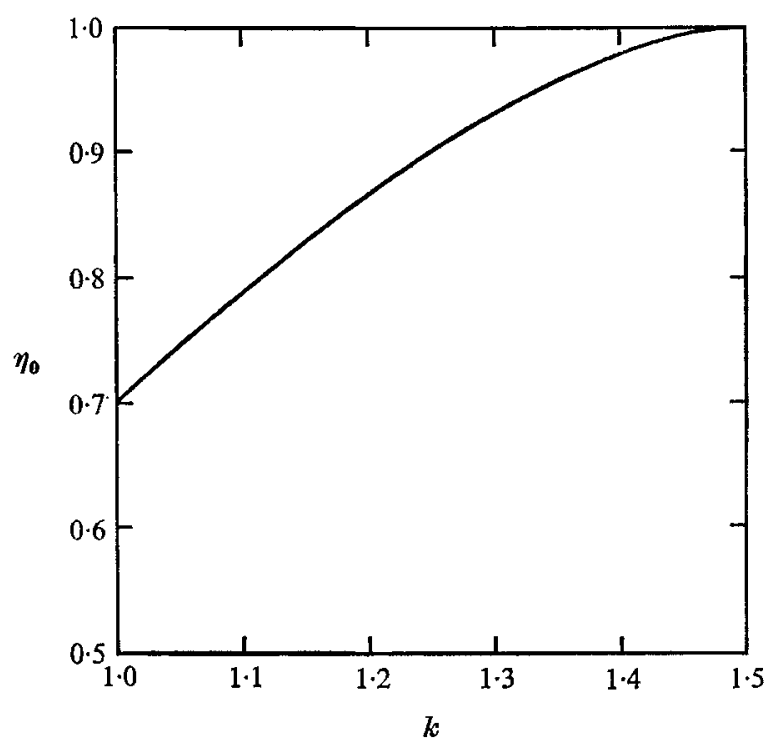

Fraure 3. Variation of the value of $\eta_{0}=\cos ^{2} \phi_{0}$ at Newtonian detachment with $k=[2+m(j-1)] / 2(1-m)$. 
The function $f$ has the correct limiting behaviours for large and small $t$. However, the constant $\beta$,

$$
\beta=\int_{0}^{\infty} \frac{d \psi}{\rho u d^{j+1}}=\frac{(1+j)^{2} \pi^{j}}{\rho_{\infty} U_{\infty} d^{1+j}} \int_{0}^{\infty} \frac{\sin ^{2} \Phi_{1}}{\cos \Phi_{1}} d \psi_{1}
$$

leads to difficulties. Writing it as $\beta=\beta_{a}+\beta_{f}$, thus dividing the integral into the regions of $\psi_{1}$ for the attached (subscript $a$ ) and free layer (subscript $f$ ) respectively gives, for a power law body,

$$
\beta_{a}=\frac{(1+j)^{2} \pi^{j}}{1-m} m^{(m j+1) /(1-m)} \int_{\Phi_{\bullet}}^{\frac{1}{2} \pi} \frac{\left(\sin \Phi_{1}\right)^{[1-(2+j) m] /(1-m)}}{\left(\cos \Phi_{1}\right)^{[2-(3+j) m] /(1-m)}} d \Phi_{1},
$$

$\Phi_{0}$ being the shock slope at the detachment point. The integral in (12) is singular at the upper limit (stagnation point) for $m<1 /(2+j)$. This singularity arises because the approximation $u=u(\psi)$ breaks down near the stagnation point. It is of the form

and

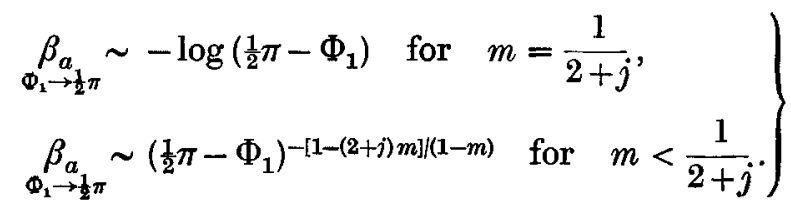

If the Bernoulli effect (see Freeman 1956, Hayes \& Probstein 1966) is included in the approximation for $u$, giving $\left[\cos ^{2} \Phi_{1}+O(\epsilon)\right]^{\frac{1}{2}}$ instead of $\cos \Phi_{1}$ in the denominator of (11), then this causes $\beta$ to be $O(\log \epsilon)$ instead of $O(1)$ in the case of $m=1 /(2+j)$. This means that it may be necessary to reconsider the form of equations (8) and (9). The contribution to $\beta$ from the free layer, $\beta_{f}$, presents no difficulty.

It will be assumed here that a more refined solution in the stagnation region will remove this difficulty, such that in principle a finite $\beta$ exists and that the asymptotic solution in the range $0<m<1 /(2+j)$ behaves as suggested by Freeman (1962b, p. 421) for general body shape.

\section{(d) Attached layer at large $x$}

Using equations (1)-(4) an expression for the attached layer thickness may be obtained in the form

$$
\begin{aligned}
& \left(\frac{y_{s}}{d}\right)^{(1+j)}-\left(\frac{x}{d}\right)^{m(j+1)}=\frac{\epsilon A}{\eta_{s}^{\frac{1}{2}}} \int_{0}^{\eta_{s}}\left(\frac{1-\eta_{1}}{\eta_{1}}\right)^{1-k} \frac{d \eta_{1}}{\eta_{1}^{\frac{s}{1}}\left[1-\eta_{s}-\frac{\frac{1}{2}\left(1-\eta_{s}\right)^{k}}{\eta_{s}^{k-\frac{s}{2}}} \int_{\eta_{\mathrm{t}}}^{\eta_{s}} \frac{\theta^{k-\frac{z}{2}}}{(1-\theta)^{k}} d \theta\right]}, \\
& \text { with } \\
& \qquad A=\frac{(1+j)^{2} \pi^{j}}{2(1-m)} m^{(1+j m)(1-m)},
\end{aligned}
$$

where the expression in square brackets is just the pressure as given by (5) on streamline 1 , and $\eta_{s}$ is $\cos ^{2} \Phi_{s}(x)$.

For small values of $\left(1-\eta_{s}\right)=\sin ^{2} \Phi_{s}$, that is, far downstream, this integral can be shown to give a layer thickness of the form

$$
\left(\frac{y_{s}}{d}\right)^{1+j}-\left(\frac{x}{d}\right)^{m(1+i)} \propto \frac{\varepsilon}{\left(1-\eta_{s}\right)^{k-1}},
$$


for half-integer values of $k$ greater than 2 , that is, for $m=3 /(4+j), 4 /(5+j)$, etc. For $m=2 /(3+j)$ the layer thickness becomes, for large $x$,

$$
\left(\frac{y_{s}}{d}\right)^{1+j}-\left(\frac{x}{d}\right)^{m(1+j)} \propto \frac{\epsilon}{1-\eta_{s}} \log \frac{1}{\left(1-\eta_{s}\right)} .
$$

The occurrence of this logarithmic singularity is interesting because it coincides in the value of $m$ with the one which is to be expected in the similar solutions of Lees \& Kubota (1957) (see Guiraud et al. 1965, p. 127, Hornung 1967a). The form of the equation for the shock shape may be obtained from (15) by writing $\left(1-\eta_{s}\right)=\sin ^{2} \Phi_{s} \doteqdot\left(d y_{s} / d x\right)^{2}$ for large $x$. This gives a differential equation which is satisfied by

$$
\frac{y_{s}}{d}=C(m, j)\left(\frac{x}{d}\right)^{m}
$$

giving similar body and shock shapes. This result has been obtained previously in the Newtonian limit by Cole (1957) who carried the solution to a higher approximation according to hypersonic small-disturbance theory. The difference here is, that similar body and shock shapes are obtained only when $m>2 /(3+j)$, which fits into the pattern of the similar solutions of Lees \& Kubota (1957) and of the blast wave solution precisely.

When $k=\frac{3}{2},(m=1 /(2+j)$ the equation of a streamline (suffix 2) comes out in the form

$$
\frac{y_{s}^{j+1}-y_{b}^{j+1}}{d^{j+1}}=\frac{\epsilon A}{\eta_{s}^{\frac{1}{2}}\left(1-\eta_{s}\right)^{\frac{3}{2}}} \log \left(\frac{\eta_{s}}{\eta_{2}}\right),
$$

again for $\left(1-\eta_{s}\right) \rightarrow 0$. This result is logarithmically singular at $\eta_{2} \rightarrow 0$ which is, of course, the same difficulty as that encountered earlier with $\beta_{a}$ at $m=1 /(2+j)$.

The above results for the layer thickness were verified by solving the integral in (14) numerically for $k=3,2,1.75$ and $\mathbf{1 . 5}$, where the singularity in (18) was avoided by starting the integration from $\eta_{2}=0.0001$. The resulting asymptotic behaviour as $\eta_{s} \rightarrow 1$ (see figure 4 ) was as predicted by equations (15), (16) and (18).

By using a test introduced by Freeman (1960) these results could be compared with experiments. At large $x$, the layer thickness $T$ for $k=\frac{3}{2}$ is approximately related to the pressure on the surface by

$$
p_{b} \propto T^{-1},
$$

as can be seen by obtaining (18) with $\eta_{2}$ small but finite and the body pressure as $p_{b} \propto\left(1-\eta_{s}\right)^{\frac{3}{2}}$. Figure 5 shows $\left(p_{b}-p_{\infty}\right) / p_{\infty}$ plotted against $T$ using the experimental results of Hornung (1967a) obtained on a two-dimensional parabola at $M_{\infty}=8.2$ in air. It is seen that this result agrees approximately with experiment.

The above results can be classified into flow types by plotting the exponent of the shock shape $y_{s}=C x^{m}$ against the exponent $m$ of the body shape. This is done in figure 6 which is to scale for axisymmetric flow. However, labelled values of $m_{s}$ and $m$ are correct for both plane and axisymmetric flow. This figure shows the three basic flow types occurring in the Newtonian limit on power law bodies:

For $2 /(3+j)<m<1$ the Newtonian layer remains attached and the shock wave and body shapes are similar for all $x$. 
For $0<m<1 /(2+j)$ the Newtonian shock layer detaches to form a free layer and the transition to the blast wave obtained by Freeman (1962b) applies, provided that a singularity arising in the stagnation region can be removed.

For $1 /(2+j)<m<2 /(3+j)$ the shock layer remains attached, but the asymptotic shock-wave shape is not similar to that of the body.

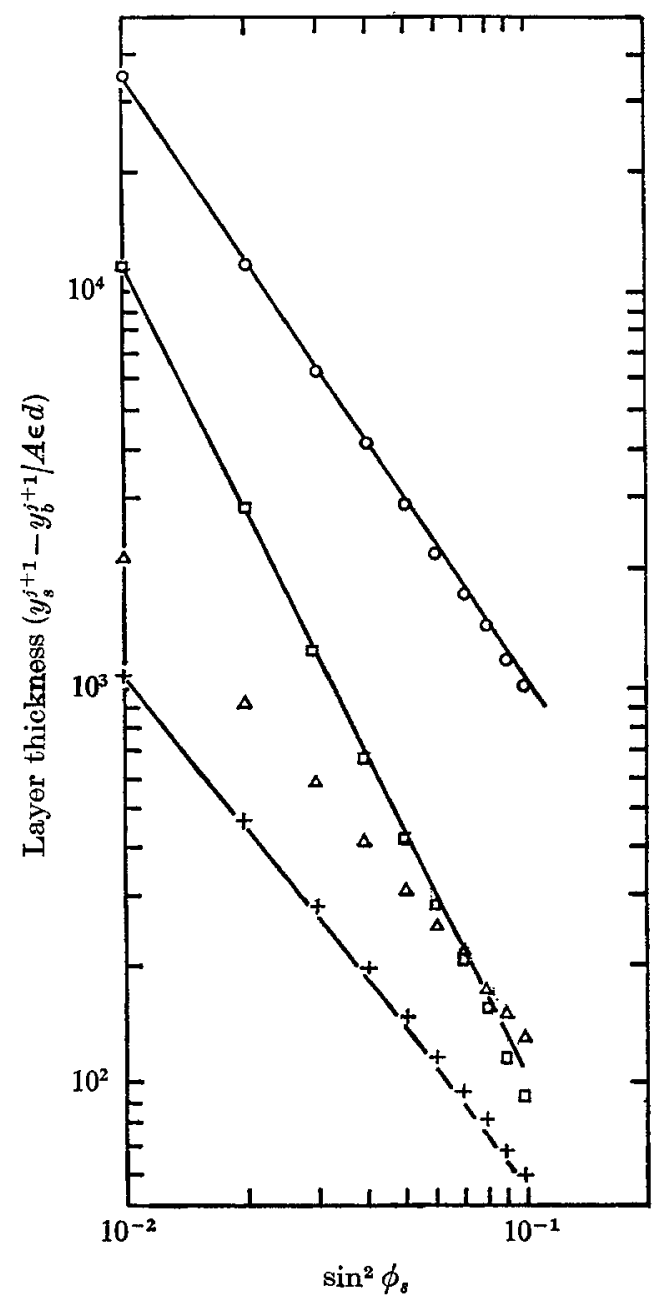

Figure 4. Numerical results for layer thickness in comparison with the form of the approximate results (-, equations (15) and (16)). $O, k=1.5 ; \square, k=3 ; \Delta, k=1.75$; ,$+ k=2$.

\section{Blast wave limit of hypersonic flow over axisymmetric power law bodies}

(a) Introduction

When $M_{\infty} \rightarrow \infty$ and $x \rightarrow \infty$, the assumption of a strong bow shock wave gives the well-known similar solutions of Lees \& Kubota (1957) for $m>2 /(3+j)$ and the Sedov-Taylor solution for $m \leqslant 2 /(3+j)$. These are first-order solutions in 


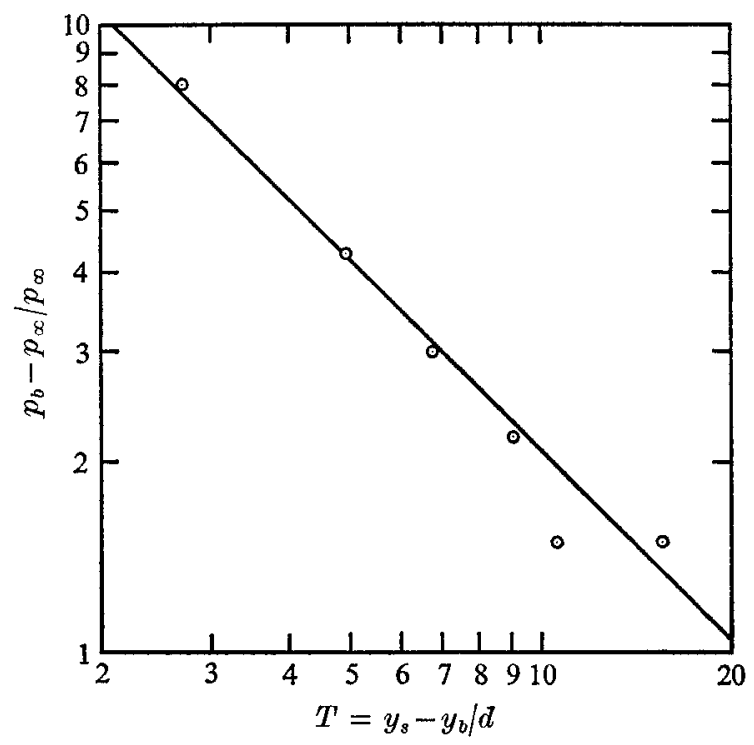

FigURe 5. Comparison of layer thickness with experiment. $\odot$, experiment, $M_{\infty}=8 \cdot 2$, $\gamma=1.4$ on plane parabola.,$-\left(p_{b}-p_{\infty}\right) / p_{\infty} \propto 1 / T$.

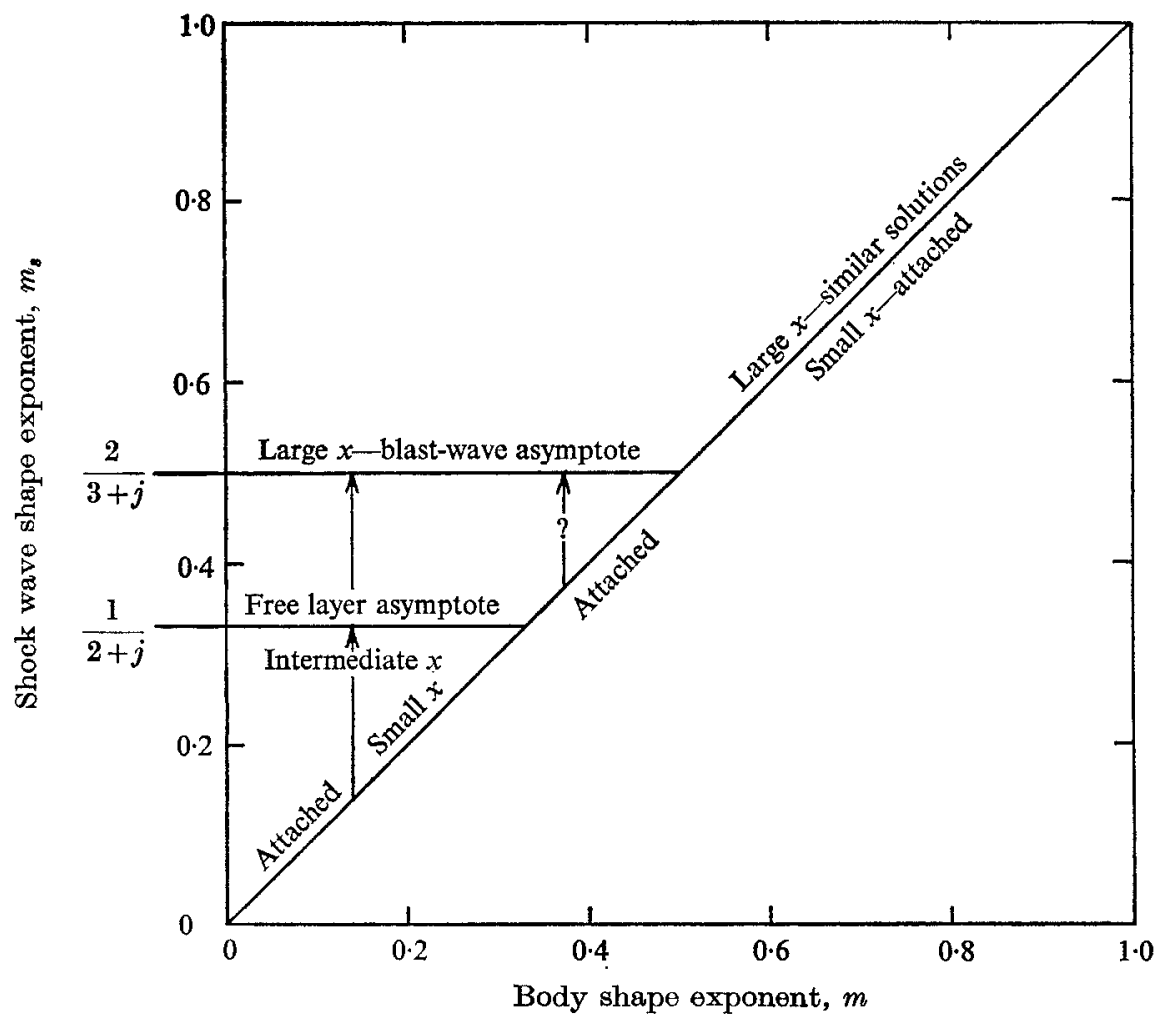

Fraure 6. Schematic diagram of flow types (to scale for axisymmetric flow). 
asymptotic expansions for large $x$, the second-order term being due to the body or the entropy layer, depending on the value of $m$. Freeman (1962 $a$ ) has shown that, whenever the entropy layer is the cause of the second-order term, a logarithmic term arises in the solution, and matching with the outer or blast wave layer becomes impossible. Guirand (1964) avoids this difficulty by requiring the coefficient of the troublesome term to be zero. In the latter approach the entropy layer grows much less rapidly, and the relative importance of body and entropy layer is altered somewhat. There has been much discussion of this problem in the literature (see, for example, Zolver 1964, Guiraud et al. 1965, Messiter 1965) but no entirely satisfactory solution has been found. More recently, Stewartson \& Thompson (1968) used a numerical technique to determine the coefficient of the logarithm term for the case $m=0$ and found that it is so small as to be zero within the error of the technique.

The only range of $m$ where this matching difficulty does not arise in the secondorder problem is $2 /(3+j) \gamma<m<2 /(3+j)$. This represents the region where the first-order solution is no longer capable of satisfying the tangency condition at the body surface (as it is for $m>2 /(3+j)$ ), and where the entropy layer term is small compared with that due to the body. The first correction term has been calculated by Hornung (1967a) for the plane case and by Hornung (1967c) for the axisymmetric case. The aim of this section is to compare the axisymmetric solutions with numerical solutions as well as with some experiments at $M_{\infty}=7 \cdot 5$ (Hornung \& Campbell 1968).

\section{(b) Asymptotic solutions}

The asymptotic solutions for axisymmetric power law bodies in the range $1 / 2 \gamma<m<\frac{1}{2}$ are very similar to those of the plane case for $2 / 3 \gamma<m<\frac{2}{3}$ and need only be outlined.

The flow variables are expanded in the form

$$
\left.\begin{array}{rl}
\frac{p}{\rho_{\infty} a_{\infty}^{2}} & =C_{0}^{2}\left(\frac{x}{d}\right)^{-1} P_{0}(\eta)\left[1+\frac{C_{1}}{C_{0}}\left(\frac{x}{d}\right)^{2 m-1} \frac{P_{1}}{P_{0}}(\eta)+\ldots\right], \\
\frac{\rho}{\rho_{\infty}} & =R_{0}(\eta)\left[1+\frac{C_{1}}{C_{0}}\left(\frac{x}{d}\right)^{2 m-1} \frac{R_{1}}{R_{0}}(\eta)+\ldots\right], \\
\frac{v}{a_{\infty}} & =C_{0}\left(\frac{x}{d}\right)^{-\frac{1}{2}} V_{0}(\eta)\left[1+\frac{C_{1}}{C_{0}}\left(\frac{x}{d}\right)^{2 m-1} V_{1}(\eta)+\ldots\right], \\
\frac{y}{\bar{V}} & =C_{0}\left(\frac{x}{d}\right)^{\frac{1}{2}} Y_{0}(\eta)\left[1+\frac{C_{1}}{C_{0}}\left(\frac{x}{d}\right)^{2 m-1} \frac{Y_{1}}{Y_{0}}(\eta)+\ldots\right],
\end{array}\right\}
$$

where $a_{\infty}$ is the free-stream sound-speed, $\eta$ is different from the $\eta$ in $\S 1$ and is defined as $\eta=\psi / C_{0}^{2} x d \rho_{\infty} U_{\infty}$, and $\psi$ is the stream function. Thus $\eta=1$ on the first-order shock wave, which has the shape $y_{s} / d=C_{0}(x / d)^{\frac{1}{2}} \cdot C_{0}$ and $C_{1}$ are functions of $m$ and $\gamma$ only and are assumed to be of order one.

The first term in the expansion (20) represents the Sedov solution and the second term is the perturbation due to the body. The body has the shape

$$
\frac{y_{b}}{d}=\left(\frac{x}{d}\right)^{m} \text {. }
$$


If (20) is substituted into the hypersonic small-disturbance equations, in which terms of order ( $\left(/ M^{2}\right)$ have already been neglected, two sets of ordinary differential equations are obtained by collecting terms of equal order. If the RankineHugoniot conditions at the shock wave are expanded as Taylor series in $\left(\eta_{s}-1\right)$ the shock-boundary conditions for these equations may similarly be separated. The tangency condition at the body is satisfied by adjusting the constant $C_{1} C_{0}$.

\begin{tabular}{|c|c|c|c|c|c|c|c|c|c|c|c|}
\hline \multirow[b]{2}{*}{$\gamma$} & \multirow[b]{2}{*}{$I_{0}$} & \multicolumn{10}{|c|}{$I_{1}$} \\
\hline & & $m=0.48$ & $m=0.46$ & 0.45 & 0.44 & 0.42 & 0.40 & 0.38 & $0 \cdot 36$ & 0.35 & $0 \cdot 32$ \\
\hline $1 \cdot 6667$ & 0.1796 & 0.417 & - & 0.398 & - & - & 0.429 & - & - & 0.687 & 1.619 \\
\hline 1.5 & $0 \cdot 2468$ & 0.665 & - & 0.693 & - & - & 0.928 & - & - & $3 \cdot 11$ & - \\
\hline $1 \cdot 4$ & 0.3132 & 0.937 & 0.998 & - & $1 \cdot 113$ & 1.331 & $1 \cdot 791$ & $3 \cdot 121$ & $14 \cdot 5$ & - & - \\
\hline $1 \cdot 3$ & 0.4227 & $1 \cdot 428$ & $1 \cdot 632$ & - & $2 \cdot 02$ & - & $6 \cdot 26$ & - & - & - & - \\
\hline $1 \cdot 2$ & 0.6382 & $2 \cdot 560$ & $3 \cdot 426$ & - & $8 \cdot 87$ & $28 \cdot 8$ & - & - & - & - & - \\
\hline & & & TABLE & Value & f the & Arrg & an & & & & \\
\hline
\end{tabular}

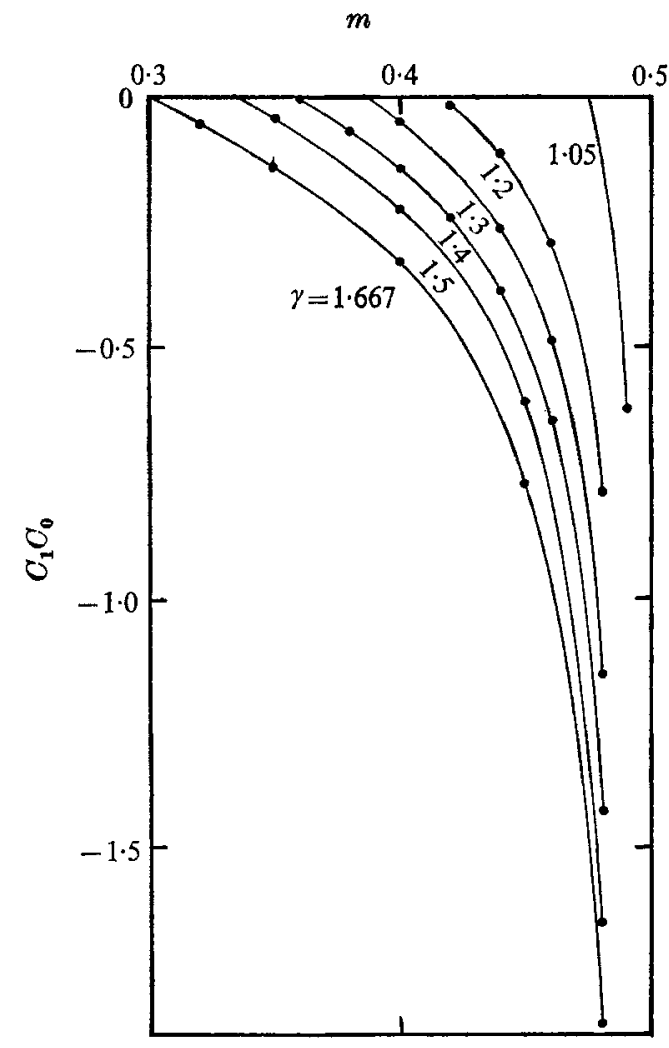

Frgure 7. Coefficient of second-order terms as a function of $m$ and $\gamma$. $(\bullet$, calculation points). 
This determines $C_{1} C_{0} . C_{0}$ cannot be obtained from the asymptotic solution but can be related to the drag, $D_{\infty}$, of an infinitely long power law body by

where

$$
\left.\begin{array}{rl}
C_{0}^{4} I_{0}=\frac{D_{\infty}}{\pi \rho_{\infty} U_{\infty}^{2} d}, \\
=\int_{0}^{1}\left[\frac{P_{0}}{(\gamma-1) R_{0}}+\frac{V_{0}^{2}}{2}\right] d \eta .
\end{array}\right\}
$$

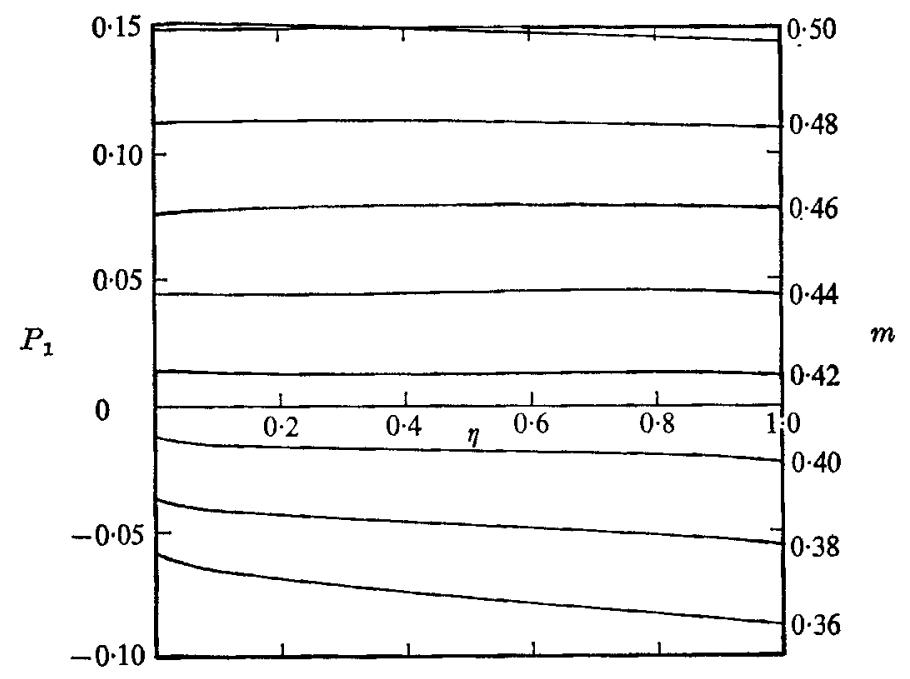

FIGURE 8. Second-order component of pressure as a function of dimensionless stream function, $\eta$, and $m$.

This is the result of an energy balance of the flow, which, in the second-order result, gives an alternative way of determining $C_{1} C_{0}$ :

where

$$
\left.\begin{array}{c}
C_{1} C_{0}\left[I_{1}+\frac{2 P_{0}(1)}{(\gamma-1) R_{0}(1)}+V_{0}^{2}(1)\right]=\frac{-2 m}{1-2 m} P_{0}(0), \\
I_{1}(m, \gamma)=\int_{0}^{1}\left[\frac{P_{0}}{(\gamma-1) R_{0}}\left(\frac{P_{1}}{P_{0}}-\frac{R_{1}}{R_{0}}\right)+V_{0} V_{1}\right] d \eta
\end{array}\right\}
$$

The integrals $I_{0}$ and $I_{1}$ are given in table 1 and $C_{1} C_{0}$ is presented graphically in figure 7.

Qualitatively these solutions are very similar to those of the plane case. An interesting feature, however, is that the second-order component of the pressure changes sign at a certain value of $m$ which depends on $\gamma$. Figure 8 shows $P_{1}(\eta)$ for $\gamma=\frac{7}{5}$ and it is seen that the value of $m$ for which $P_{1}$ is approximately zero is between 0.40 and 0.42 . In figure 9 the value of $m$ for which $P_{1}(0)$ is zero is presented as a function of $\gamma$. This shows that within the region of interest $\left(1 / 2 \gamma<m<\frac{1}{2}\right)$ there is a value of $\gamma$ below which $P_{1}(0)$ is always positive, and above which it can be negative depending on $m$. This is approximately at $\gamma=\mathbf{1 \cdot 2 6}$. Above this value of $\gamma$, there is thus a value of $m$ for which the second-order pressure is zero at the body surface. 


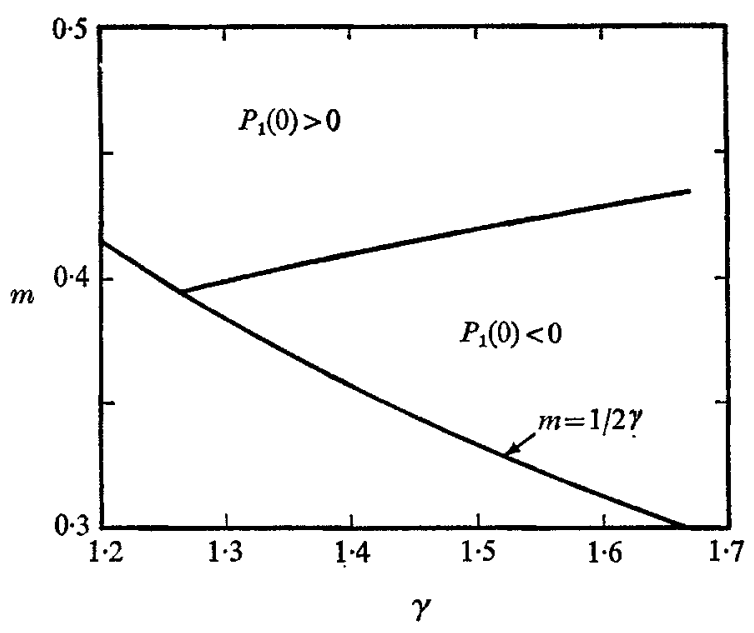

Frgure 9. The value of $m$ for which $P_{1}(0)=0$.

\section{(c) Numerical solutions}

The computer program which was used to obtain a numerical solution was made available by the NASA Ames Laboratory. It is described in detail by Lomax \& Inouye (1964). Essentially it consists of two parts, the first of which uses an inverse blunt-body technique to calculate the subsonic flow behind the bow shock wave. The shock shape is assumed known and adjustments are made to it in successive iterations to make the resulting body fit the required body shape to within specified limits. This program yields a data line in the supersonic region which, together with the remaining body shape, is then used as the input to the second part of the program which uses the method of characteristies.

The first part of the program is not able to cope with the infinite radius of curvature at the nose of power law bodies for which $m<\frac{1}{2}$. To overcome this, the body shape used in the subsonic region was an ellipse of 'best fit' which joins the power law downstream with continuous slope, and matches the ordinate of the power law at some point closer to the origin. The error in the shape was always less than $2 \%$ of the characteristic nose dimension $d$, so that the effect on the flow far downstream might be considered to be small. However, as will be seen later, this seems to have some effect on the flow near the body at large $x$.

Calculations were made at $M_{\infty}=100, \gamma=1.4$, and for $m=0.4$ and 0.44 . Because the Mach number was not infinite the strong shock-wave assumption was not exactly satisfied. At $M_{\infty}=100$ this caused the density ratio across the shock to differ from the strong shock value by about $10 \%$. In the pressure ratio the corresponding error was about $0 \cdot 3 \%$. At $x / d=30$, these figures are approximately halved.

To compare these calculations with the asymptotic theory it is necessary to determine the unknown constant $C_{0}$ from equation (21). The value of $D_{\infty}$ was obtained by determining the drag up to $x, D_{x}$, from the numerical solutions by integrating the pressure distribution, and then fitting it to

$$
D_{x}=D_{\infty}+A(x / d)^{2 m-1} \text {. }
$$


The error in $C_{0}$ determined in this way is estimated to be less than $1 \%$. For $m=0.40$ and 0.44 respectively $C_{0}$ was 1.26 and 1.40 . The pressure distribution on the body as given by the asymptotic theory using this value of $C_{0}$ is compared with the numerical results in figure 10, and a similar comparison of shock-wave shapes is given in figure 11. Figure 10 shows that the surface-pressure distribution is given quite accurately by the asymptotic theory and that the pressure does approach its asymptote from opposite sides for the two values of $m$ as predicted. The shock-wave shapes are not in such good agreement, but the numerical result lies between first-and second-order theory.

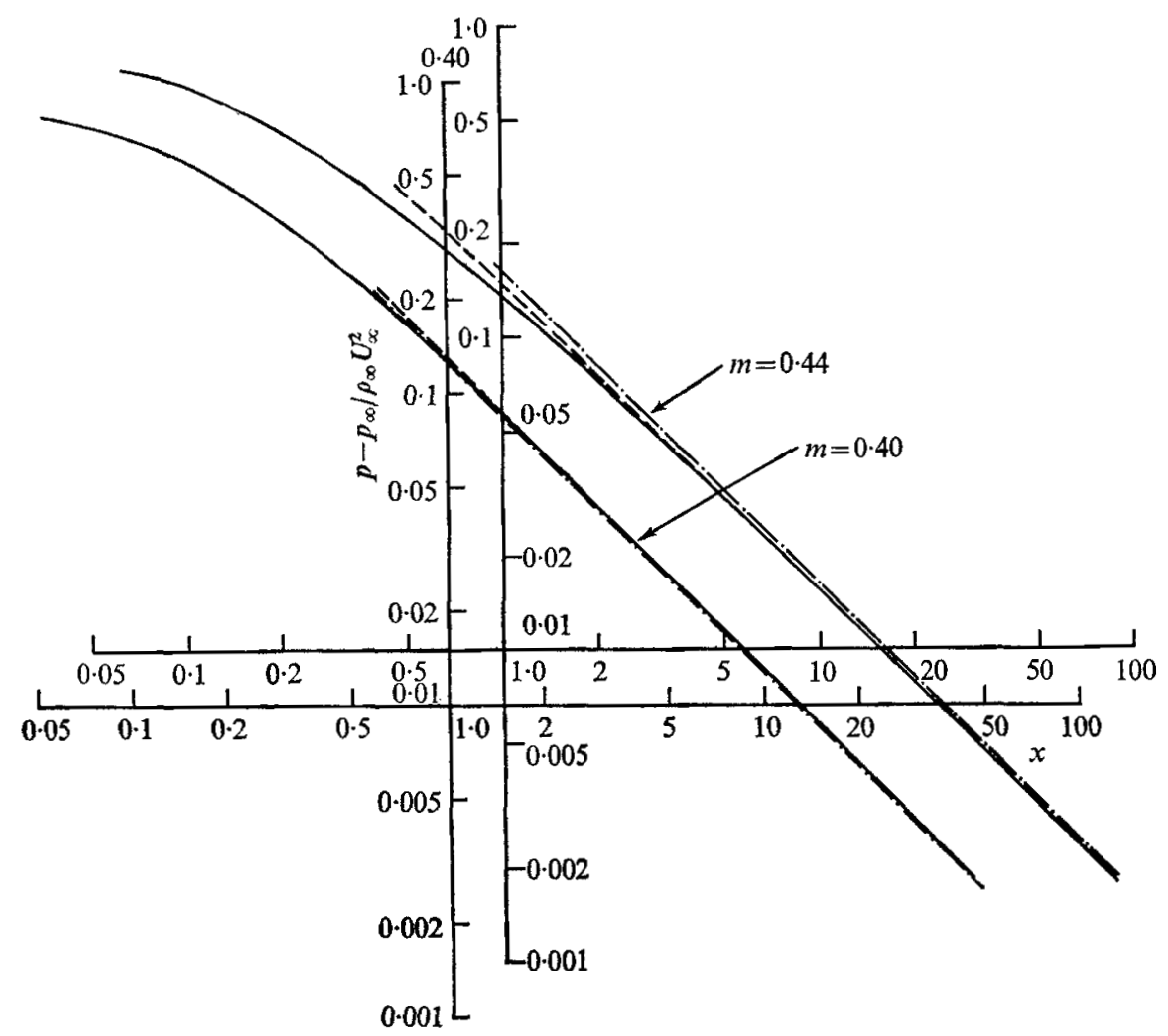

FIGURE 10. Surface pressure distribution, $\gamma=1 \cdot 4$. - , numerical calculation, $M_{\infty}=100$; -.-, first-order asymptotic theory; ---, second-order asymptotic theory. Note: $x$ is measured from the stagnation point on the elliptic cap.

Profiles of pressure, density and streamline slope across the shock layer as calculated numerically (points) are compared with asymptotic theory (lines) in figures $12(a)$ and $(b)$. With the exception of density, the sign of the deviation from first-order theory is predicted correctly by the second-order result. Its magnitude is fairly accurate in the streamline slope and, for large $x$, in the pressure. The discrepancy in density is to be expected, since the second-order theory is not capable of removing the singularity in the body boundary condition for the density inherited from the first-order result. To remove this, it would be 
necessary to include the entropy layer as a third-order effect. Figure 12 shows $p, \rho$ and $\tan \theta$, where $\theta=$ streamline slope, normalized by their values on the shock wave at the same $x$.

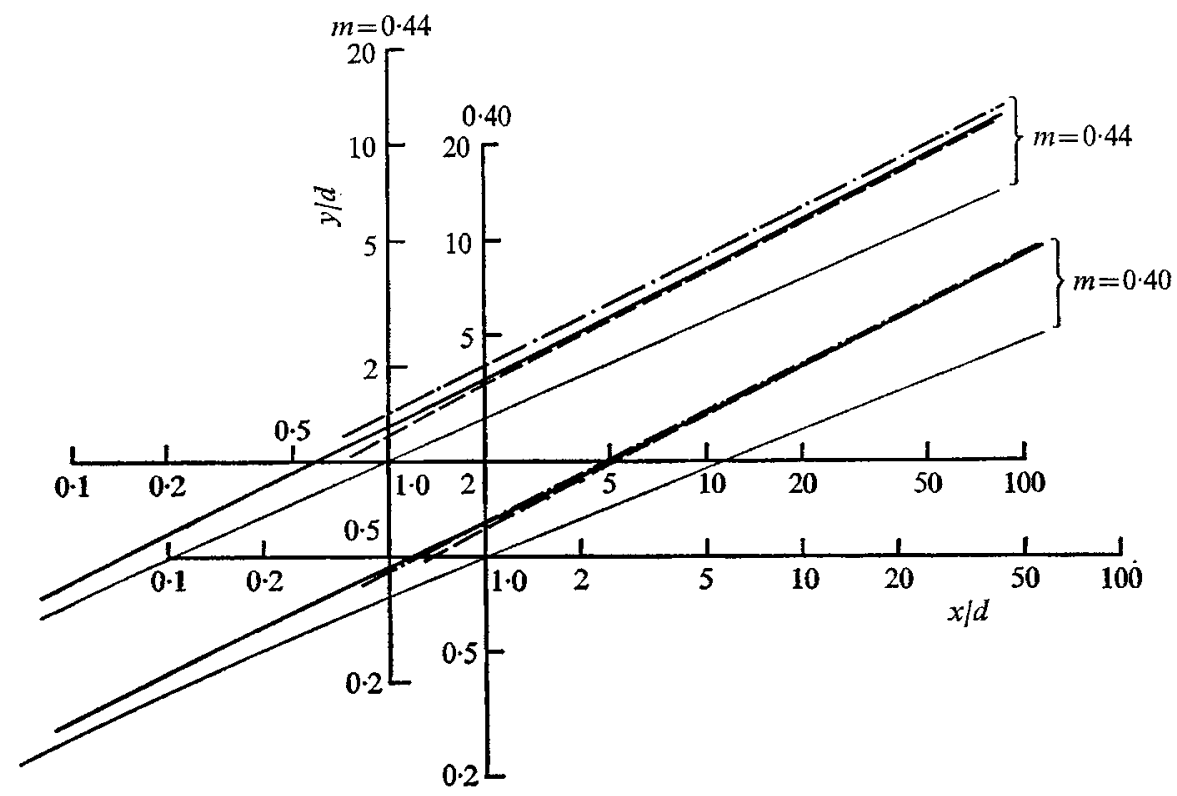

FIGURe 11. Shock-wave shape, $\gamma=1 \cdot 4$. -, numerical calculation, $M_{\infty}=100 ;-\cdot-$, firstorder asymptotic theory; --, second-order asymptotic theory; -, body shape. Note: the abscissa for the shock-wave shape has been changed here to make $x=0$ when the shock ordinate is zero. The stand-off distances are $0.0884 d$ and $0.0988 d$ for $m=0.44$ and 0.40 respectively. For the body, $x$ is as in figure 10 .

\section{(d) Comparison with experimental results}

Measurements of surface pressure, shock-wave shape and shock-layer traverses of pitot and static pressure have been made by Hornung \& Campbell (1968) on 10 axisymmetric power law models of five different powers in the range $1 / 2 \gamma<m<\frac{1}{2}$. These experiments were made in air at a free stream Mach number of $7 \cdot 55$, free stream Reynolds number of $10^{6}$ per foot (stagnation temperature $=630^{\circ} \mathrm{K}$, stagnation pressure $=125 \mathrm{psia}$ ), with the characteristic model dimension, $d$, ranging from 0.04 to $0.5 \mathrm{in}$.

In order to compare these experimental results with the asymptotic theory, it is again necessary to determine the constant $C_{0}$. This will be different from that in the numerical calculations at $M_{\infty}=100$, since it is now determined from the experimental pressure drag at $M_{\infty}=\mathbf{7 \cdot 5}$. Shock-wave shapes as measured from Schlieren photographs are compared with asymptotic theory in figure 13. This shows the experimental points together with associated first- and second-order theory for four powers, in logarithmic co-ordinates. At $m=0.5$ a theoretical result cannot be shown, since $C_{0}$ cannot be obtained, and at $m=0 \cdot 36$ the secondorder result is indistinguishable from first-order theory. The dotted lines show the result of numerical calculations using the computer programs of Lomax \& Inonye (1964) at the experimental conditions for $m=0.36,0.40$, and 0.44 . 


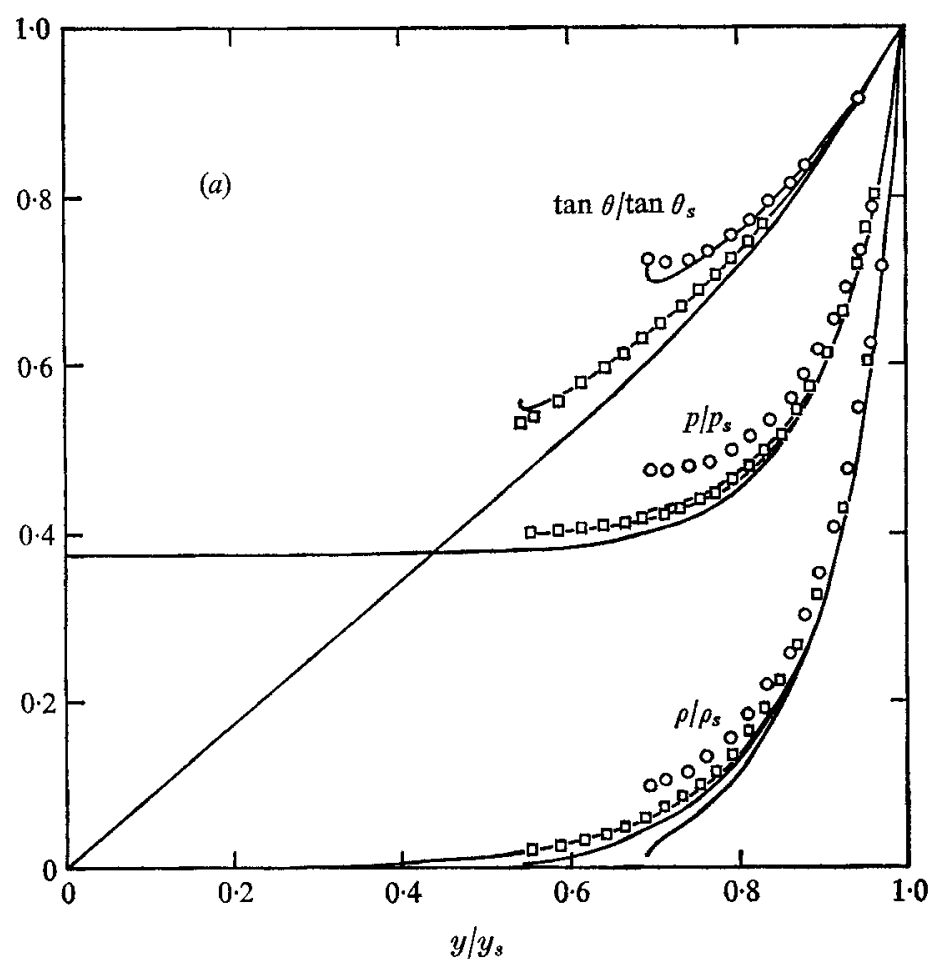

Figure 12. (a) Profiles of flow parameters across the shock layer $m=0 \cdot 40, \gamma=1.4$. -, second-order asymptotic theory for $\left(C_{1} / C_{0}\right)(x / d)^{(2 m-1)}=0,-0 \cdot 05$ and $-0 \cdot 100 . \odot, \square$, numerical solution for $M_{\infty}=100, x / d=4.57$ and 39.3 respectively. $\theta=$ streamline slope.

It is seen that the asymptotic solutions provide a reasonable estimate of the shock-wave shape over an intermediate range of $x / d$. At lower $x / d$, the assumption of the asymptotic theory break down, and at large $x / d$ the shock wave becomes weak. The latter effect manifests itself in a tendency to a straight line of slope $=$ one in logarithmic co-ordinates, rather than to the blast wave limit slope of $\frac{1}{2}$. The good agreement between numerical and experimental results for the shock-wave shape shows that the discrepancy between experiment and second-order asymptotic theory is not due to viscous displacement. It is probably due to the weak shock effect, which primarily causes the density ratio across the shock wave in the experiment to be somewhat less than $(\gamma+1) /(\gamma-1)$.

A correlation of shock-wave shapes in a transition region between blast wave and weak shock regions is likely to be of the form

$$
\frac{y_{s}}{d \sqrt{ }\left(M_{\infty}^{2}-1\right)}=f\left[\frac{x}{d\left(M_{\infty}^{2}-1\right)}\right]
$$

since this satisfies both the blast wave similarity, $\left(y_{s} / d\right)=C_{0}(x / d)^{\frac{1}{2}}$, and the Mach wave limit, $y_{s}=x / \sqrt{ }\left(M_{\infty}^{2}-1\right)$. The experimental results of Peckham (1965), Kubota (1957) and Hornung \& Campbell (1968) for $m=0.5$ were plotted in the co-ordinates of the above correlation in figure 14, and it is seen that the experimental points have a fairly narrow scatter. Unfortunately, the experimental 


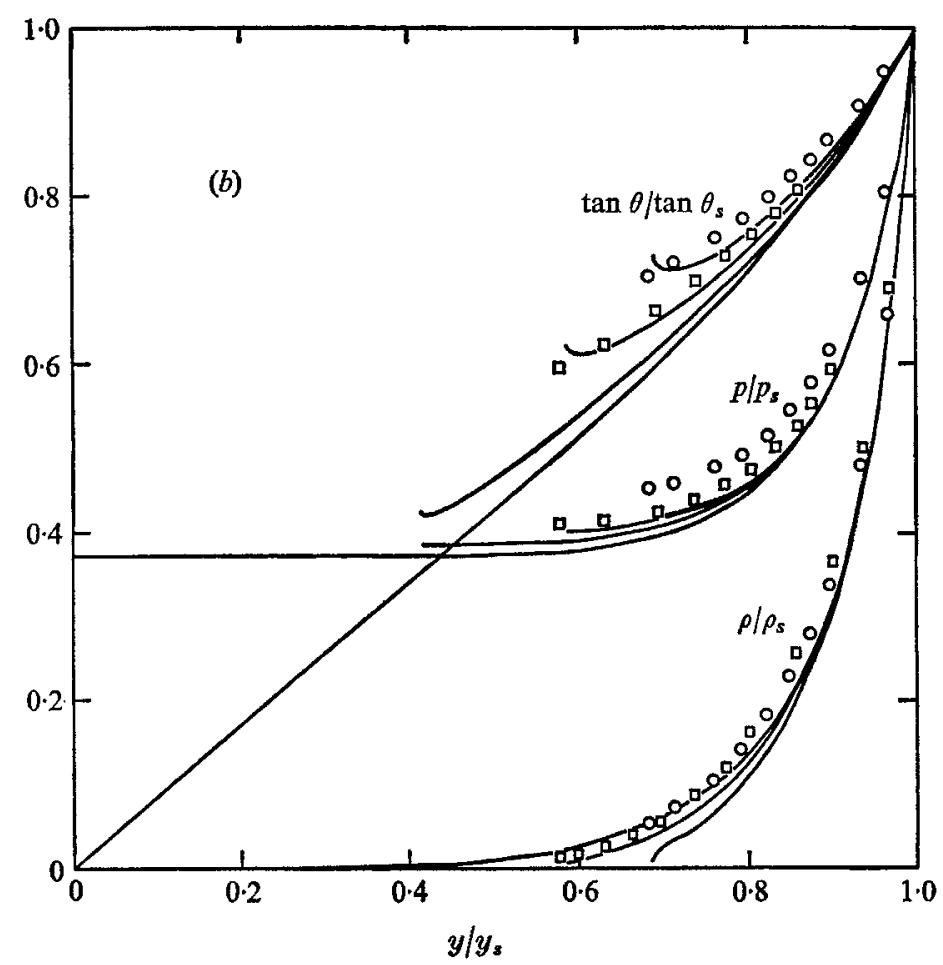

FIGURE 12. (b) $m=0.44, \gamma=1.4 .$, , second order asymptotic theory for

$$
\left(C_{1} / C_{0}\right)(x / d)^{(2 m-1)}=0,-0.0538,-0 \cdot 100 \text { and }-0.15 \text {. }
$$

$\odot, \square$, numerical calculation for $M_{\infty}=100, x / d=10.5$ and 87.7 respectively. $\theta=$ stream. line slope.

results were all obtained at similar Mach numbers so that this test is not as critical as it might have been.

Figure 15 shows a comparison of theoretical and experimental pressure distribution in logarithmic co-ordinates. By plotting these in the form $\left(p-p_{\infty}\right) / \rho_{\infty} U_{\infty}^{2}$ the weak shock effect is approximately corrected, and it is seen that agreement with asymptotic theory is quite good for the lower powers. At $m=0.44$ the numerical solution has been plotted as well. This is lower than experiment by up to about $15 \%$. This could be due to the error introduced by the fitting of the elliptic cap to the power law shape, as this involves a curvature discontinuity at the junction of ellipse and power law. In part, of course, it is due to boundary layer growth. It is impossible to distinguish between first- and second-order theory for $m=0.36$ and 0.40 in figure 15 , so that the change in sign of $P_{1}(0)$ from $m=0.4$ to 0.44 cannot be detected. However, this result is consistent with the theoretical one that the surface pressure should be given by first-order theory exactly at $m=0 \cdot 41$.

Shock layer traverses of pitot and static pressure are shown in figure $16(a)$ and $(b)$ for $m=\mathbf{0} \cdot 40$. Similar results are given for the other powers by Hornung $\&$ Campbell (1968). The abscissa in these traverses is $z / d$ were $z$ is the distance measured normal to the body surface at the traversing station. The 


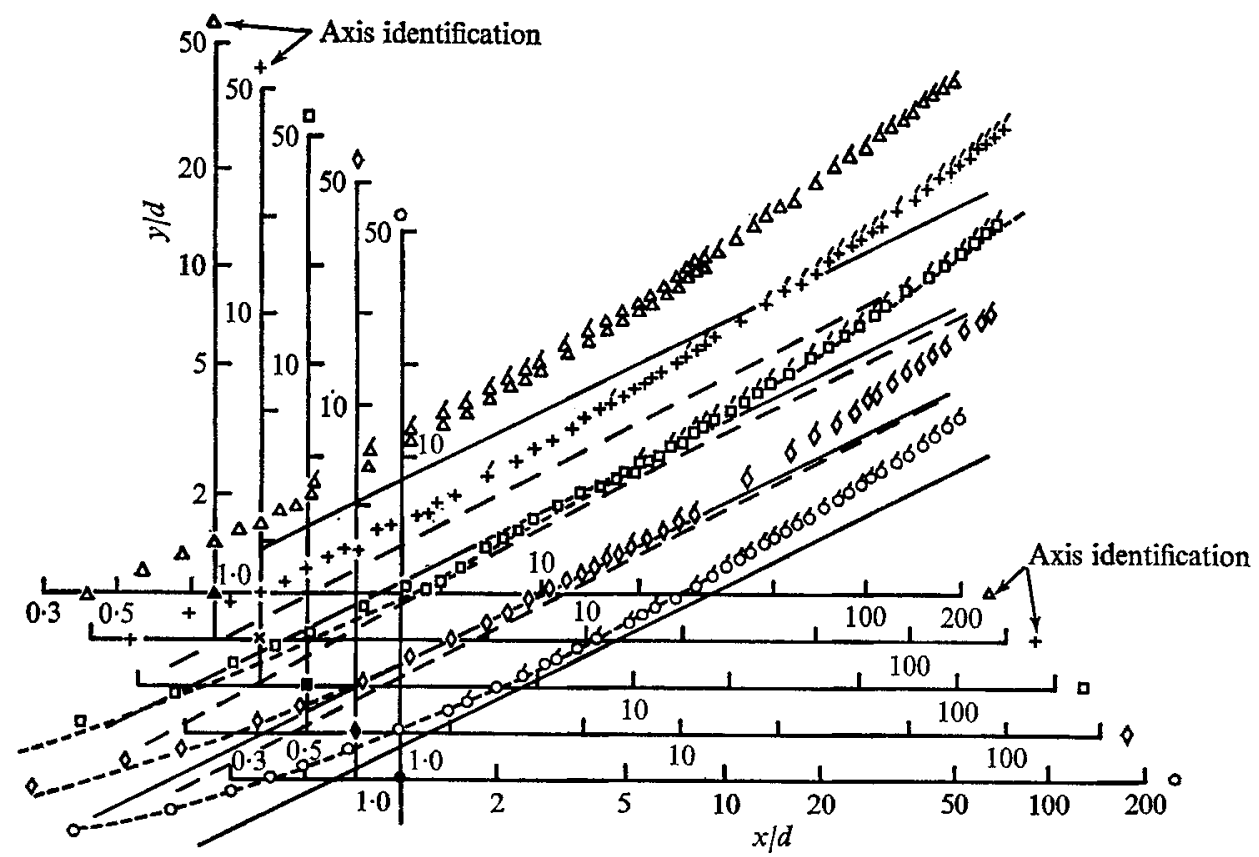

FIGURE 13. Shock-wave shape compared with experiment $M_{\infty}=7 \cdot 55$. 一, first-order asymptotic theory; ---, second-order asymptotic theory; ..., numerical calculation. Experiment: $O, \delta, m=0.36, d=0.494,0.123$ in.; $\diamond, \diamond, m=0.40, d=0.368,0.092$ in.; $\square$, , $, m=0.44, d=0.263,0.066$ in.; $+, f, m=0.48, d=0.179,0.045$ in.; $\Delta, \triangle$, $m=0.50, d=0.144,0.036$ in.

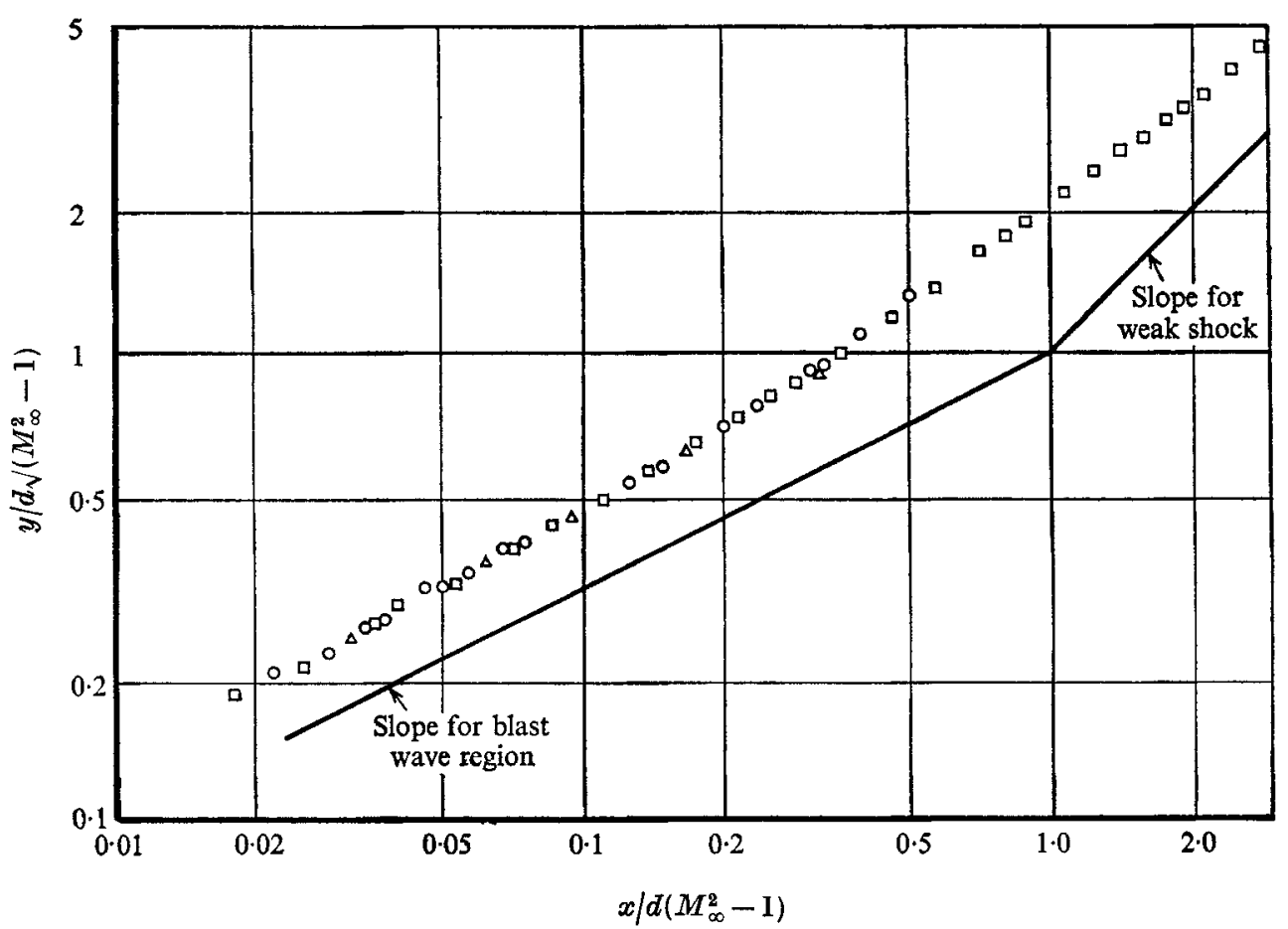

FIGURE 14. Correlation of shock-wave shapes at finite Mach number, $m=0.5$. $\odot$, Peckham (1965), $M_{\infty}=6.85 ; \Delta$, Kubota (1957), $M_{\infty}=7.7 ; \square$, present results, $M_{\infty}=7.55$. 
static-pressure traverses are seen to compare well with surface pressure and shock slope at the same station. Traverses from the numerical calculations are also shown.

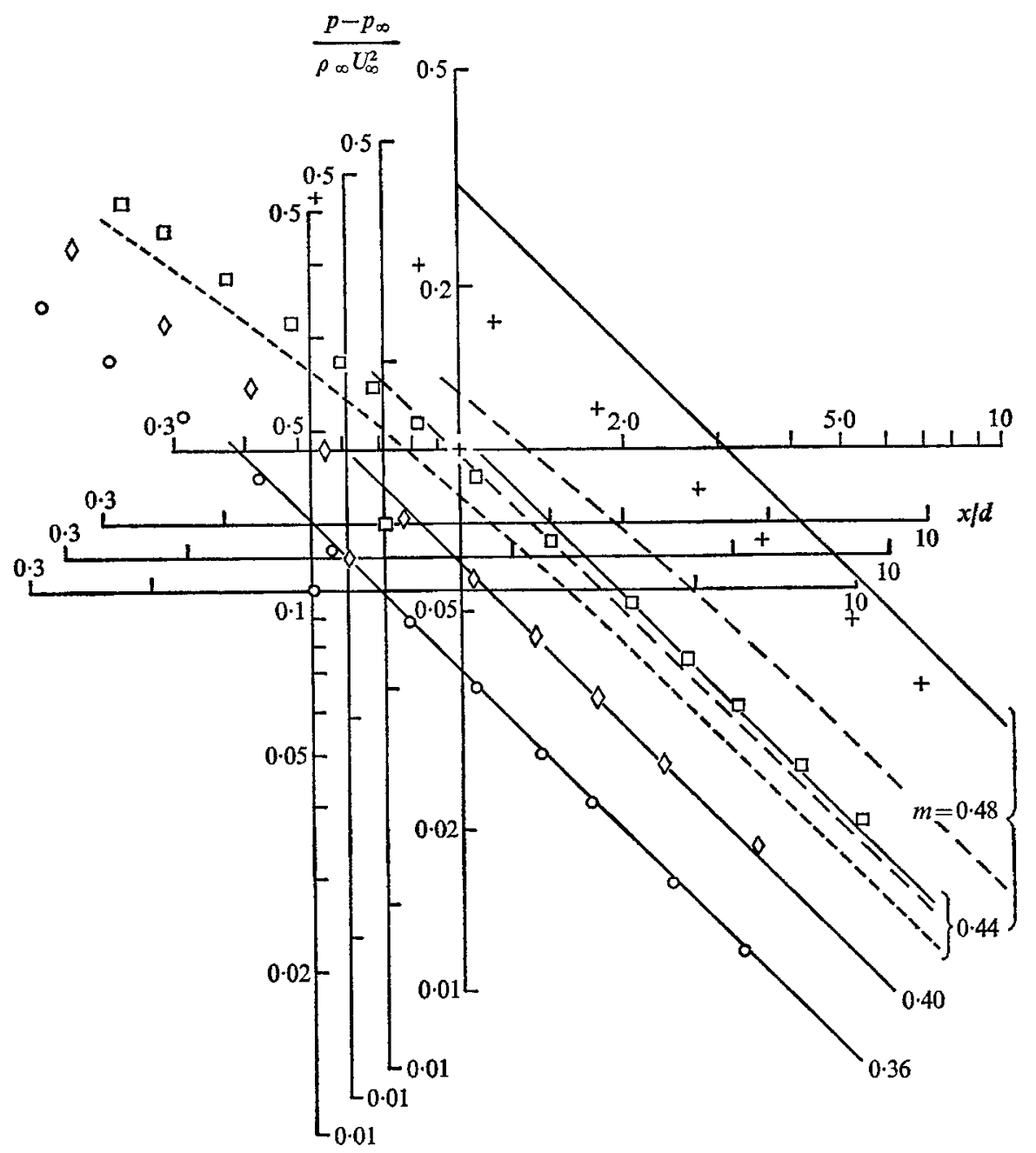

Figure 15. Surface pressure distribution compared with experiment. - , first-order asymptotic theory; ---, second-order asymptotic theory; ..., numerical calculation. Experiment:,$+ m=0 \cdot 48 ; \square, m=0.44 ; \diamond, m=0 \cdot 40 ; 0, m=0 \cdot 36$.

The static and Pitot pressure profiles are concave upwards at low values of $x / d$ as in the asymptotic theory, and become progressively less so further downstream. This is the result of the weak shock effect which reduces the pressure and density at the shock wave. The Pitot pressure traverses are continuous as they were taken by transducer and $X, Y$ plotter. They show the extent of the boundary layer, but because the probe thickness was too large, the distribution through the boundary layer is probably considerably in error. The outside dimension of the probes is shown to scale on both figures $16(a)$ and $(b)$. 


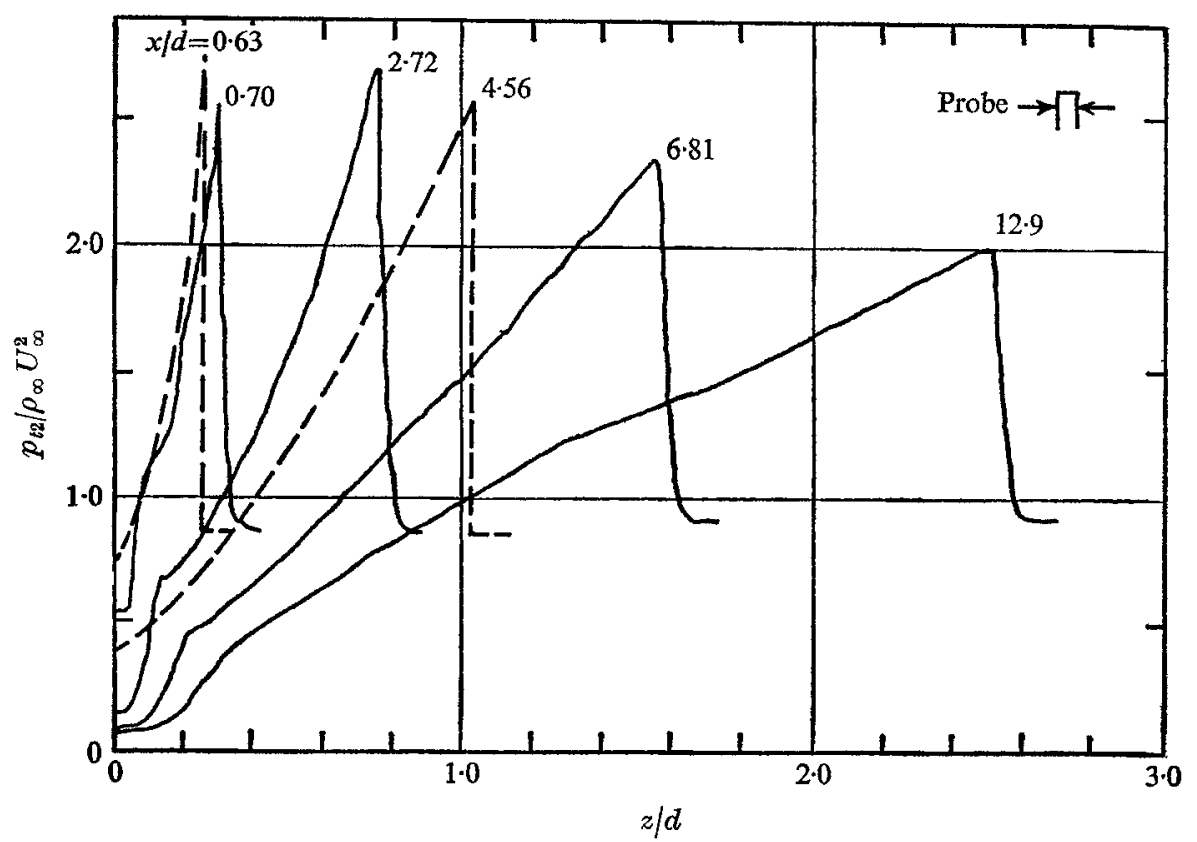

(a)

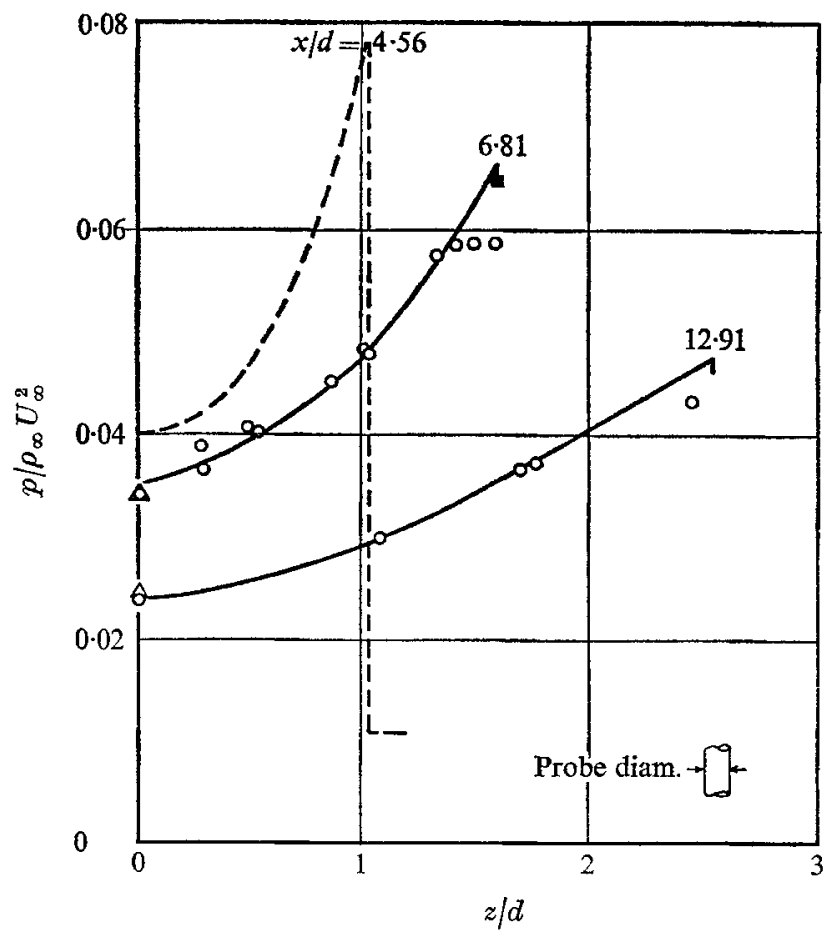

(b)

Figure 16. (a) Pitot pressure distribution $\left(p_{t 2}\right)$ across shock layer, $m=0 \cdot 40$;, , experiment; - -, numerical calculation. (b) Static pressure distribution across shock layer, $m=0 \cdot 40 . \ominus$, static probe; $\Delta$, surface pressure; $\mathbf{E}$, from shock wave slope; ---, numerical calculation. 
Most of this work was done at the Aeronautical Research Laboratories, Department of Supply, Australia. The help of the Aerophysics Group is gratefully acknowledged and thanks are due to the Chief Superintendent for permission to publish.

\section{REFERENCES}

COLE, J. D. 1957 J. Aero. Sci. 24, 448.

Fremman, N. C. 1956 J. Fluid Mech. 1, 366.

Freeman, N. C. $1960 \mathrm{~J}$. Fluid Mech. 8, 109.

Fremman, N. C. 1962a NPL Aero. 1035, ARC 23999, Hyp. 265. (See also Research Frontiers in Fluid Dynamics, ed. Seegar and Temple. New York: Interscience.)

Freeman, N. C. $1962 b$ Hypersonic Flow Research. (Ed. F. R. Ridell.) New York: Academic.

GuIraud, J. P. 1964 Int. Symp. on Fluid Phenomena in Hypersonic Flow, Buffalo. Cornell University Press.

Guiraud, J. P., VAILet, D. \& Zolver, R. 1965 Basic Developments in Fluid Dynamics, vol. I, (ed. M. Holt). New York: Academic.

Hayes, W. D. \& Probsters, R. F. 1966 Hypersonic Flow Theory, vol. I. New York: Academic.

HoRnUng, H. G. 1967 a J. Fluid Mech. 27, 315.

Hornung, H. G. $1967 b$ ARL Aero. Note 287 Dept. of Supply, Australia.

Hornung, H. G. 1967 c ARL Aero. Note 288 Dept. of Supply, Australia.

Hornung, H. G. \& Campbeil, I. A. 1968 ARL Aero. Rep. 130, Australia.

Kubota, T. 1957 GALCIT Memo. no. 40.

LeEs, L. \& Kubota, T. 1957 J. Aero. Sci. 24, 195.

LighthiLl, M. J. 1957 J. Fluid Mech. 2, 1.

Lomax, H. \& INouYe, M. 1964 N.A.S.A. TR R-104.

Messiter, A. F. 1965 B.A.M.I.R.A.C. Rep. no. 4613-81-T Michigan University.

Peckham, D. 1965 Aero. Res. Coun. 27084.

Stewartson, K. \& Thompson, B. W. 1968 Proc. Roy. Soc. A 304, 255.

Zolver, R. 1964 C. R. Acad. Sci., Paris, 258, 5152. 